\title{
Combination of Soft X-Ray Microscopy with In-Situ Mechanical Testing to Image Crack Propagation in Microchips
}

Kristina Kutukova ${ }^{1, *}$, Zhongquan Liao ${ }^{1}$, Stephan Werner $^{2}$, Peter Guttmann ${ }^{2}$, Yvonne Standke ${ }^{1}$, Jürgen Gluch $^{1}$, Gerd Schneider ${ }^{2}$, Ehrenfried Zschech ${ }^{1}$

${ }^{1}$ Fraunhofer Institute for Ceramic Technologies and Systems, Maria-Reiche-Straße 2, 01109 Dresden, Germany

${ }^{2}$ Helmholtz-Zentrum Berlin, Albert-Einstein-Straße 5, 12489 Berlin, Germany

* kristina.kutukova@ikts.fraunhofer.de

The combination of high-resolution X-ray imaging with in-situ mechanical testing, particularly the application of a specially designed indenter manipulator in the full-field transmission X-ray microscope at the U41-PGM1-XM beamline of the synchrotron radiation source BESSY II [1] (see Fig. 1a), is used to study the fracture behavior of microchips. The X-ray microscope was operated at a photon energy of $1200 \mathrm{eV}$ for the experiments described here. The achieved spatial resolution of about $25 \mathrm{~nm}$ allows to image the metal interconnect structures in microchips manufactured in advanced technology nodes. The mechanical manipulation of the samples was performed using a Picoindenter PI95 (Bruker) (Fig. 1b), equipped with a tungsten wedge indenter (Fig. 1c) [2].

Thermomechanical stress in microchips increases the risk of failure in on-chip interconnect stacks, also called backend-of-line (BEoL) stack [3], caused by delamination along $\mathrm{Cu}$ /dielectrics interfaces (adhesive failure) and/or fracture in dielectrics (cohesive failure). In this paper, crack propagation in the BEoL stack of a microprocessor manufactured in an advanced technology node is presented. It consists of 12 layers of $\mathrm{Cu}$ interconnects which are insulated by thin film materials with low dielectric permittivity (low-k materials) [4]. Particularly designed dense metal structures, so-called crack stop structures, are supposed to dissipate energy and to slow down or even stop the crack propagation [5]. Such structures are visualized in Figure 2 [6]. A lamella sample with the region of interest (BEoL stack of the microchip with crack stop structures) was thinned to a thickness of $2.5 \mu \mathrm{m}$ using the focused ion beam (FIB) technique, to get high absorption contrast in the X-ray images. The sample was prepared in such a way that the tungsten wedge indenter could be applied to initiate the crack and to propagate the crack while increasing the load.

Figure 3 demonstrates the sequence of crack evolution [2]. The initial crack $(12 \mu \mathrm{m}$ length and $225 \mathrm{~nm}$ width) was positioned at the cross-sectioned BEoL structure (scribe line position which is the location where micro-cracks can be initiated during the wafer dicing process) towards the crack stop structure between metal layer 8 and metal layer 9 (Fig. 3a). Fig. 3b shows the crack which is stopped in front of the crack stop structure. Subsequently, the crack propagates between metal layer 8 and metal layer 7, and finally, it is directed to metal layer 4 (Fig. 3c). The example demonstrates that the pathway of cracks, and consequently the weakest structures for cracking, can be identified in fully integrated, realistic multilevel $\mathrm{Cu} /$ low-k interconnect structures of microchips manufactured in advanced technology nodes.

In summary, the application of a novel, specially designed indenter manipulator in a transmission X-ray microscope allows the (in-situ) visualization of crack opening and propagation in $\mathrm{Cu} / \mathrm{low}-\mathrm{k}$ interconnect stacks of microchips, and to distinguish between adhesive and cohesive failure. These studies provide data regarding the robustness of BEoL stacks against thermomechanical stress and for the evaluation of the effectiveness of the design of crack-stop structures [7]. 


\section{References:}

[1] S. Rehbein et al, Optics Express 20, 5830-5839 (2012)

[2] K. Kutukova et al, MRS Advances, submitted (2018)

[3] A. Grill et al, Appl. Phys. Rev. 1, 011306 (2014)

[4] S. Natarajan et al, IEEE International Electron Devices Meeting (IEDM), December 2014, San Francisco/CA (2014)

[5] X. Zhang et al, AIP Conf. Proc. 1143, 197 - 203 (2009)

[6] K. Kutukova et al, International FCMN conference, March 2017, Monterey/CA (2017)

[7] The authors thank Oden Warren and Douglas Stauffer (both with Bruker Corporation, Billerica/MA, USA) as well as Martin Gall, Andre Clausner and Christoph Sander (all with Fraunhofer IKTS Dresden, Germany) for helpful discussions. Financial support from SRC under Member-Specific Research Contract P30697 is greatly appreciated.

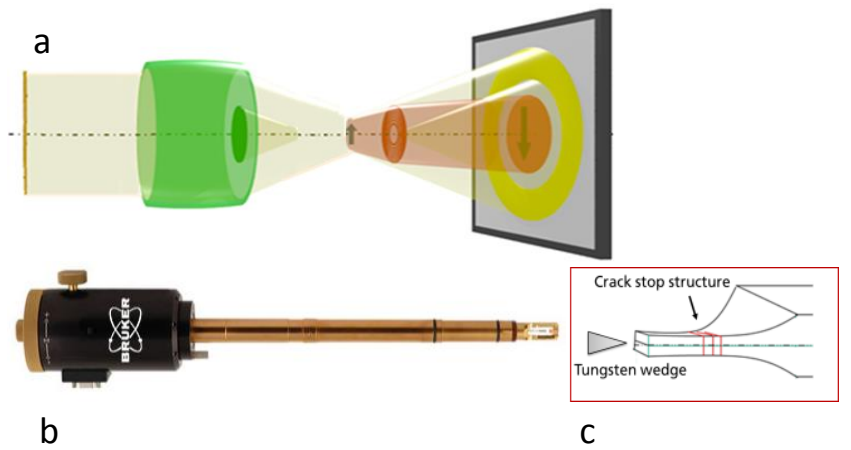

Figure 1. (a) Optical set-up of the full-field transmission X-ray microscope: Monochromatized synchrotron radiation, condenser with central stop, sample with mechanical test setup, Fresnel zone plate lens, CCD as detector. (b) Mechanical manipulator (Picoindenter PI95, courtesy Bruker Corporation). (c) Scheme of the indentation of the BEoL stack using a tungsten wedge [2].

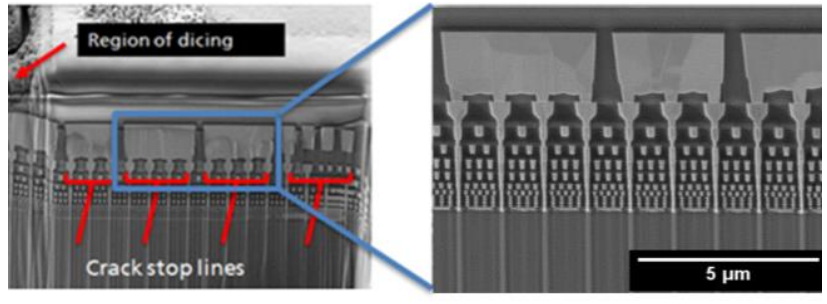

Figure 2. SEM cross-section images with crack stop structures of a leading edge microprocessor with 12 layers of $\mathrm{Cu}$ interconnects [6].

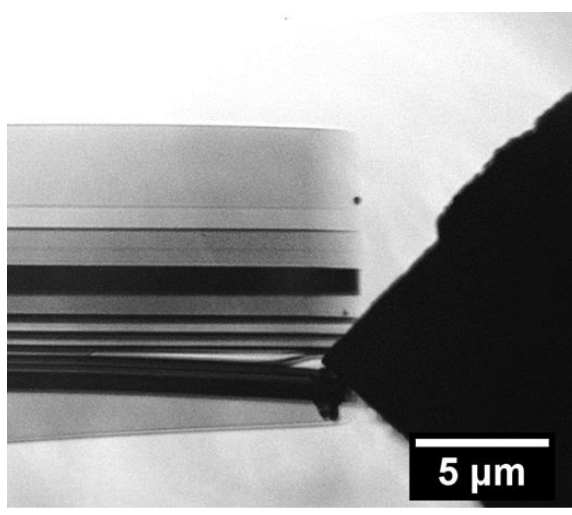

a

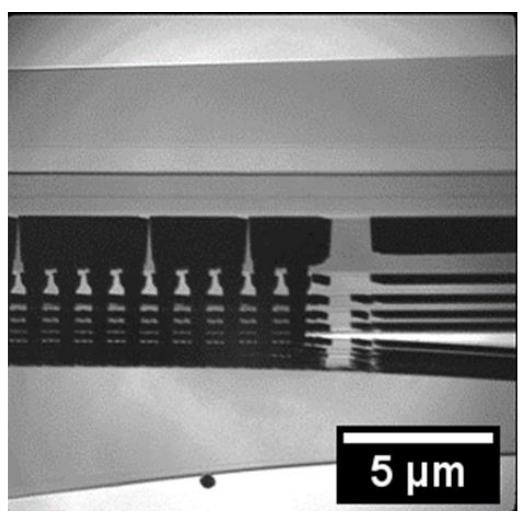

b

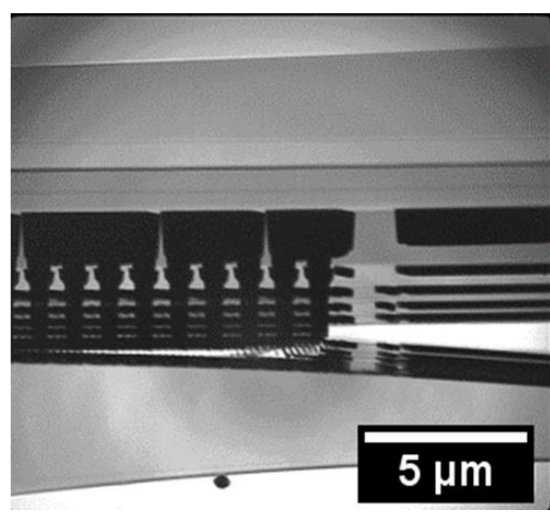

c

Figure 3. (a) The tungsten wedge indenter is initiating the crack from in the scribe line (toward the crack stop). (b) The crack is stopped in front of the crack stop structure, then it propagated down to metal 8. (c) The crack propagates between metal 8 and metal 7, and is then directed to metal 4 [2]. 